\title{
Suppression of Fibrotic Reactions of Chitosan-Alginate Microcapsules Containing Porcine Islets by Dexamethasone Surface Coating
}

\author{
Min Jung Kim ${ }^{1}$, Heon-Seok Park ${ }^{1}$, Ji-Won Kim ${ }^{1}$, Eun-Young Lee ${ }^{1}$, Marie Rhee ${ }^{1}$, Young-Hye You ${ }^{1}$, Gilson Khang ${ }^{2}$, \\ Chung-Gyu Park ${ }^{3}$, Kun-Ho Yoon ${ }^{1,4}$ \\ ${ }^{1}$ Department of Endocrinology and Metabolism, College of Medicine, The Catholic University of Korea, Seoul; ${ }^{2}$ Department of \\ Polymer Nano Science and Technology, Department of BIN Fusion Technology and BK-21 Polymer BIN Fusion Research \\ Team, Chonbuk National University, Jeonju; ${ }^{3}$ Department of Microbiology and Immunology, Translational Xenotransplantation \\ Research Centre, Cancer Research Institute, Biomedical Research Institute, Seoul National University College of Medicine; \\ ${ }^{4}$ Department of Endocrinology and Metabolism, Seoul St. Mary's Hospital, College of Medicine, The Catholic University of \\ Korea, Seoul, Korea
}

Background: The microencapsulation is an ideal solution to overcome immune rejection without immunosuppressive treatment. Poor biocompatibility and small molecular antigens secreted from encapsulated islets induce fibrosis infiltration. Therefore, the aims of this study were to improve the biocompatibility of microcapsules by dexamethasone coating and to verify its effect after xenogeneic transplantation in a streptozotocin-induced diabetes mice.

Methods: Dexamethasone 21-phosphate (Dexa) was dissolved in 1\% chitosan and was cross-linked with the alginate microcapsule surface. Insulin secretion and viability assays were performed 14 days after microencapsulation. Dexa-containing chitosan-coated alginate (Dexa-chitosan) or alginate microencapsulated porcine islets were transplanted into diabetic mice. The fibrosis infiltration score was calculated from the harvested microcapsules. The harvested microcapsules were stained with trichrome and for insulin and macrophages.

Results: No significant differences in glucose-stimulated insulin secretion and islet viability were noted among naked, alginate, and Dexa-chitosan microencapsulated islets. After transplantation of microencapsulated porcine islets, nonfasting blood glucose were normalized in both the Dexa-chitosan and alginate groups until 231 days. The average glucose after transplantation were lower in the Dexa-chitosan group than the alginate group. Pericapsular fibrosis and inflammatory cell infiltration of microcapsules were significantly reduced in Dexa-chitosan compared with alginate microcapsules. Dithizone and insulin were positive in Dexa-chitosan capsules. Although fibrosis and macrophage infiltration was noted on the surface, some alginate microcapsules were stained with insulin. Conclusion: Dexa coating on microcapsules significantly suppressed the fibrotic reaction on the capsule surface after transplantation of xenogenic islets containing microcapsules without any harmful effects on the function and survival of the islets.

Keywords: Diabetes mellitus; Islets of Langerhans transplantation; Dexamethasone; Cell encapsulation; Fibrosis

Received: 22 October 2020, Revised: 30 October 2020,

Accepted: 3 November 2020

Corresponding author: Kun-Ho Yoon

Department of Endocrinology and Metabolism, Seoul St. Mary's Hospital, College of Medicine, The Catholic University of Korea, 222 Banpo-daero, Seocho-gu, Seoul 06591, Korea

Tel: +82-2-2258-6901, Fax: +82-2-595-2534, E-mail: yoonk@catholic.ac.kr

\section{Copyright $(2021$ Korean Endocrine Society}

This is an Open Access article distributed under the terms of the Creative Commons Attribution Non-Commercial License (https://creativecommons.org/ licenses/by-nc/4.0/) which permits unrestricted non-commercial use, distribution, and reproduction in any medium, provided the original work is properly cited. 


\section{INTRODUCTION}

Type 1 diabetes is an autoimmune disease resulting from $\beta$-cell destruction, in which patients are incapable of maintaining euglycemia without insulin injection [1,2]. Islet transplantation is an alternative method of exogenous insulin that can allow insulin secretion depending on glucose level $[3,4]$. Since the introduction of the Edmonton protocol based on immunosuppressants of daclizumab, sirolimus, and tacrolimus, human islet transplantation has increased $[5,6]$. However, the graft failure rate remains high at up to $90 \%$ at 5 years after transplantation [7]. In addition, immunosuppressants could have an adverse effect on islet function and survival $[8,9]$. Treatment with tacrolimus and sirolimus reduces islet size and induces islet apoptosis in rats [8]. In humans, these drugs disrupt insulin granule formation and insulin processing via cleavage at dibasic sites and upregulate amyloid deposition [9].

One of the promising methods to avoid immune attack after islet transplantation is to microencapsulate islets to protect islets from immune response-mediated destruction. Microencapsulation can allow penetration of small molecules, such as oxygen, nutrients, and insulin, while preventing pass-through of high molecular weight components, such as immunoglobulin and macrophages [10]. Many researchers have studied capsular materials, such as alginate, collagen, and silica. Among them, alginate microcapsules are a common technique based on poly-Llysine used to protect transplanted islet grafts [11,12]. However, this approach has a limitation of fibrosis on the microcapsule surface, which is induced by the immune response of the host via macrophages and small molecule antigens. The fibrotic reaction on the capsular surface influences the permeability of the capsules and thus inhibits nutrient and oxygen exchange. This reaction induces hypoxia and destruction of encapsulated islets, leading to necrosis of islets and graft failure [13].

To improve biocompatibility and subsequently reduce fibrosis associated with microcapsules, various anti-inflammatory drugs have been applied as a strategy to minimize host response and enhance the stability of transplantable microcapsules [14,15]. Dexamethasone 21-phosphate (Dexa) is a commonly used antiinflammatory drug that potently suppresses inflammatory pathways. Dexa has been reported to suppress the immune reaction of transplanted devices by selective suppression of pro-inflammatory factors, such as macrophages [16]. Dexa also prevents the proliferation of fibroblasts in mice [17] and pulmonary fibrosis induced by bleomycin [18]. However, high-dose systemic administration of Dexa could impair islet function and survival
[19-21]. In contrast, a low dose of Dexa improves islet survival and preserves islet revascularization [13].

Therefore, we aimed to improve the biocompatibility and fibrosis of microcapsules by Dexa surface coating on alginate.

\section{METHODS}

\section{Animals}

The Seoul National University (SNU) designated pathogen free (DPF) pigs aged at least 6 months were used as xenogenic islet donors [22]. Male alpha 1, 3-galactosyltransferase knock-out mice (GalT KO) aged 7 to 12 weeks (kindly provide from Prof. Chung-Gyu Park, SNU) were used as recipients. Type 1 diabetes was confirmed with a portable glucometer (GLUCOCARD X-METER, Arkray, Kyoto, Japan); if random blood glucose levels from the tail vein exceeded $300 \mathrm{mg} / \mathrm{dL}$ for three consecutive days after intraperitoneal one-shot injection of $200 \mathrm{mg} / \mathrm{kg}$ dose of streptozotocin (STZ, Sigma-Aldrich, St. Louis, MO, USA; freshly dissolved in $0.01 \mathrm{M}$ citric acid buffer, $\mathrm{pH} 4.5$ ), the diagnosis of type 1 diabetes was made. The GalT KO mice were maintained at a constant temperature in a cycle of 12 hours of light followed by 12 hours of dark and provided with laboratory chow and water. All of surgical interventions and presurgical and postsurgical animal care were provided in accordance with the Laboratory Animals Welfare Act, the Guide for the Care and Use of Laboratory Animals and the Guidelines and Policies for Rodent Survival Surgery provided by the IACUC (Institutional Animal Care and Use Committee) in College of Medicine, The Catholic University of Korea (approval number: CUMS-20140105-02).

\section{Materials}

Pronova ultrapure low viscosity guluronic acid (UP-LVG) sodium alginate (20 to $200 \mathrm{kDa}$ ) was purchased from Pronova (Novamatrix, Sandvika, Norway). Povidone was purchased from Sungkwangpham (Cheonan, Korea). Acridine orange (AO), magnesium sulfate $\left(\mathrm{MgSO}_{4}\right)$, magnesium chloride $\left(\mathrm{MgCl}_{2}\right)$, sodium phosphate monobasic $\left(\mathrm{NaH}_{2} \mathrm{PO}_{4}\right)$, sodium chloride $(\mathrm{NaCl}$ ), chitosan (from crab shells, minimum $85 \%$ deacetylated), barium chloride $\left(\mathrm{BaCl}_{2}\right)$, nicotinamide, 4-(2-hydroxyethyl) piperazine-1-ethanesulfonic acid (HEPES), propidium iodide (PI), STZ, luria/miller agarose (LB agarose), paraformaldehyde (PFA), fluorescein isothiocyanate (FITC)-labeled dextran, sodium hydroxide $(\mathrm{NaOH}), \mathrm{H}_{2} \mathrm{O}_{2}$, potassium chloride $(\mathrm{KCl})$, calcium chloride $\left(\mathrm{CaCl}_{2}\right)$, gluconic acid, potassium hydroxide $(\mathrm{KOH})$, L-histidine, D-mannitol, sodium pyruvate, monobasic potassi- 
um phosphate $\left(\mathrm{KH}_{2} \mathrm{PO}_{4}\right)$, citric acid, 4'-6-diamidino-2-phenylindole (DAPI), hydrochloride (HCl), sodium bicarbonate (NaH$\mathrm{CO}_{3}$ ), 3,3'-diaminobenzidine (DAB), harris hematoxylin, dithizone (DTZ), mayer's hematoxylin, biebrich scarlet aqueous, acid fuchsin, glacial acetic acid, phosphotungstic acid, light green, and OptiPrep ${ }^{\mathrm{TM}}$ were purchased from Sigma Aldrich. Dulbecco's phosphate-buffered saline (DPBS), RPMI 1640, Media 199 (M199), fetal bovine serum (FBS), antibiotic/antimycotic solution, gentamycin, and porcine serum (PS) were obtained from GIBCO (Carlsbad, CA, USA). Dexa was purchased from Jeil pharm (Seoul, Korea). Cefazolin was purchased from ChongKunDang (Seoul, Korea), ketamine HCl from Yuhan Corporation (Seoul, Korea), Clzyme collagenase MA and CIzyme BP protease from VitaCyte (Indianapolis, IN, USA) and Phenol red free $1 \times$ Hank's balanced salt solution $(1 \times$ HBSS; without $\mathrm{Ca}^{2+}, \mathrm{Mg}^{2+}$ ) was purchased from WelGENE Inc. (Gyeongsan, Korea).

\section{Islet isolation procedure}

Porcine islets were isolated from SNU DPF adult pigs according to established protocols $[22,23]$. After cannulation of the pancreatic duct using cold histidine-tryptophan-ketoglutarate (HTK) solution (CustodiolTM, Koehler Chemi, Bensheim, Germany), the pancreas was delivered in cold HTK solution. The delivered pancreas was washed sequentially with $1 \%$ cold povidone, a cold solution of antibiotics $(0.2 \%$ cefazolin, $0.016 \%$ gentamycin dissolved in DPBS), and cold DPBS. Washed pancreas was trimmed to remove non-pancreatic tissues in cold HTK solution, then CIzyme collagenase MA $(0.12 \mathrm{CDA} /$ pancreas gram)/BP ( 0.03 million neutral protease activity units per pancreas gram) protease in $200 \mathrm{~mL}$ of phase I solution (10 mM $\mathrm{NaOH}, 5.8 \mathrm{mM} \mathrm{KOH}, 1 \mathrm{mM} \mathrm{CaCl} 2,1.6 \mathrm{mM} \mathrm{MgSO}_{4}, 45 \mathrm{mM}$ D-mannitol, $28.8 \mathrm{mM} \mathrm{NaH}_{2} \mathrm{PO}_{4}, 105 \mathrm{mM} \mathrm{NaCl}$ ) was injected into the pancreatic duct. Injected pancreas was digested in a Ricordi chamber (Biorep technologies, Miami Lakes, FL, USA) and $500 \mathrm{~mL}$ phase I solution addition, where it was incubated without shaking for 20 to 25 minutes at $35^{\circ} \mathrm{C}$ to $37^{\circ} \mathrm{C}$ until the pancreatic tissue was loosened, followed by gentle shaking and serial sampling. When free islet cells were observed in the sample, digestion was stopped by adding $20 \%$ PS in cold phase II solution $(90 \mathrm{mM}$ gluconic acid, $30 \mathrm{mM}$ L-histidine, $20 \mathrm{mM}$ $\mathrm{KOH}, 0.3 \mathrm{mM} \mathrm{CaCl}_{2}, 10.2 \mathrm{mM} \mathrm{MgSO}_{4}, 50 \mathrm{mM}$ D-mannitol, 5 $\mathrm{mM}$ sodium pyruvate, $20 \mathrm{mM} \mathrm{KH}_{2} \mathrm{PO}_{4}$ ). Digested pancreatic tissues were collected and washed in $10 \%$ PS in cold phase II solution and centrifuged for 2 minutes at 1,000 rpm (Hanil science industrial, Gimpo, Korea). Islets were purified using a continuous OptiPrep ${ }^{\mathrm{TM}}$ density gradient $(1.100,1.085$, and $1.060 \mathrm{~g} / \mathrm{cm}^{3}$ density) and a Cobe 2991 cell separator (Gambro BCT Inc., Lakewood, CO, USA). The purified islets were washed with cold phase II solution and cultured in M199 supplemented with $10 \%$ PS, $16 \mathrm{mM}$ nicotinamide, $24 \mathrm{mM} \mathrm{NaH}-$ $\mathrm{CO}_{3}$ and $1 \%$ penicillin/streptomycin at $37^{\circ} \mathrm{C}$ in humid conditions with $5 \% \mathrm{CO}_{2}$.

\section{Islet microencapsulation procedure}

Alginate solution for microencapsulation was prepared that 2\% (w/v) UP-LVG alginate was dissolved in $\mathrm{Ca}^{2+}$-free Krebs-Ringer-HEPES buffer (KRH buffer: $135 \mathrm{mM} \mathrm{NaCl}, 4.7 \mathrm{mM} \mathrm{KCl}$, $25 \mathrm{mM}$ HEPES, $1.2 \mathrm{mM} \mathrm{KH}_{2} \mathrm{PO}_{4}, 1.2 \mathrm{mM} \mathrm{MgSO}_{4}$ ). After 1 to 2 days in cultured, the isolated SNU DPF porcine islets were suspended in a solution of $2 \%$ UP-LVG alginate and at a concentration of $10,000 \mathrm{IEq} / \mathrm{mL}$ for SNU DPF porcine islets. Microcapsules were made with a coaxial bead generator (Nisco Engineering Inc., Zürich, Switzerland) that expelled islet-solution mixture using a syringe pump (Harvard apparatus, Holliston, MA, USA) into a $150 \mathrm{~mm}$ petri-dish (SPL, Pocheon, Korea) of $10 \mathrm{mM} \mathrm{BaCl}_{2}$ solution ( $10 \mathrm{mM} \mathrm{BaCl}, 2 \mathrm{mM} \mathrm{KCl}, 135$ $\mathrm{mM} \mathrm{NaCl}, 10 \mathrm{mM}$ HEPES, $\mathrm{pH}$ 7.4). The encapsulated islets were washed with $1 \times \mathrm{HBSS}$ and $1 \mathrm{mg} / \mathrm{mL}$ Dexa contained $\mathrm{pH}$ $4.51 \%$ filtrated chitosan solution (w/v in deionized distilled water) for 10 minutes with stirring for Dexa-chitosan coating. Chitosan, high affinity positive charged residue [24], was used as an intermediary to link alginate and Dexa because both alginate and Dexa have negative charges.

All these procedures are made at room temperature. After chitosan coating, microcapsules were washed 3 to 4 times with $1 \times$ HBSS and cultured in M199 supplemented with 10\% PS, 16 $\mathrm{mM}$ nicotinamide, $24 \mathrm{mM} \mathrm{NaHCO} 3$ and $1 \%$ penicillin/streptomycin at $37^{\circ} \mathrm{C}$ in humid conditions with $5 \% \mathrm{CO}_{2}$.

\section{Confirm of microcapsules morphology and pore size}

Dexa-chitosan-coated alginate surface was observed using transmission electron microscopy (TEM; JSM-1010, JEOL Ltd., Tokyo, Japan). Microcapsule pore size was determined by the diffusion FITC-labeled dextran conjugates of various sizes [25]. Alginate and Dexa-chitosan-coated alginate microcapsules were incubated for 3 days in $1 \times$ HBSS with FITC-labeled dextran conjugates $(4,10,20,40,70,150,250 \mathrm{kDa})$. After incubating, each microcapsule was imaged under a confocal fluorescence microscope (AxioVert 200TM, Carl Zeiss, Oberkochen, Germany). 


\section{In vitro dexamethasone 21-phosphate release test}

The standard curve was produced by dissolving Dexa in $1 \times$ HBSS; a range of 0 to $500 \mu \mathrm{g} / \mathrm{mL}$ was obtained. The standard Dexa concentration was measured using a UV/Vis spectrometer (Nanodrop, Thermo Fisher Scientific, Waltham, MA, USA) at an absorption wavelength of $240 \mathrm{~nm}$. The 1,000 ea Dexa-chitosan-coated alginate microcapsules were incubated in a $100 \mathrm{~mm}$ petri-dish with $10 \mathrm{~mL} 1 \times \mathrm{HBSS}$ solution, at $37^{\circ} \mathrm{C}$ under humid conditions with $5 \% \mathrm{CO}_{2}$, for the Dexa release test. After 35 days, $10 \mu \mathrm{L}$ supernatant samples were obtained from the incubated petri-dish and measured using the same UV/Vis wavelengths as the standard preparation spectrometer [26]. A regression analysis of the in vitro Dexa release was used to determine the following equation for the Dexa standard curve: $Y=[0.412 \times$ $\left.\mathrm{X}\left(R^{2}=1.000\right)\right]$, where $\mathrm{Y}$ is the concentration of Dexa $(\mu \mathrm{g} / \mathrm{mL})$, $\mathrm{X}$ is the value of the absorbency of Dexa, and $R^{2}$ is the regression coefficient.

\section{Microencapsulated islets viability measurement}

The standard AO/PI method was used to evaluate islet viability $0,1,3,5,7$, and 14 days after microencapsulation. Naked, alginate microencapsulated, and Dexa-chitosan-coated alginate microencapsulated islets were incubated in $1 \times$ HBSS with $1 \%$ FBS with AO/PI for 10 minutes. Viability of these islets was evaluated using AO/PI double staining to observe viable (AO; green) and dead cells (PI; red) with an inverted fluorescence microscope (Observer.Z1, Carl Zeiss).

\section{Glucose stimulated insulin secretion test}

Tests were performed on islet samples of $100 \mathrm{IEq}$ (150 to 250 $\mu \mathrm{m}$ diameter islet selection) for each group. Islet samples were transferred to a $60 \mathrm{~mm}$ petri-dish (SPL) and incubated in a $5 \mathrm{~mL}$ 1\% FBS contained glucose-free Krebs-Ringer-Bicarbonate buffer (KRB buffer: $24 \mathrm{mM} \mathrm{NaHCO}_{3}, 5 \mathrm{mM} \mathrm{KCl}, 115 \mathrm{mM} \mathrm{NaCl}$, $2.5 \mathrm{mM} \mathrm{CaCl}_{2}, 1 \mathrm{mM} \mathrm{MgCl} 2,25 \mathrm{mM}$ HEPES) for 1 hour at $37^{\circ} \mathrm{C}$ in humid conditions with $5 \% \mathrm{CO}_{2}$ before performing the glucose-stimulated insulin secretion (GSIS) test. Then, each group of islets was incubated in a $5 \mathrm{~mL} \mathrm{1 \%} \mathrm{FBS} \mathrm{contained} \mathrm{low-}$ glucose $(2.8 \mathrm{mM}) \mathrm{KRB}$ buffer for 1 hour $37^{\circ} \mathrm{C}$ in humid conditions with $5 \% \mathrm{CO}_{2}$. After incubation, each group of islets was washed with an 1\% FBS contained glucose-free KRB buffer and incubated in a $5 \mathrm{~mL} \mathrm{1 \%} \mathrm{FBS} \mathrm{contained} \mathrm{high-glucose} \mathrm{(16.8}$ $\mathrm{mM}) \mathrm{KRB}$ buffer for 1 hour at $37^{\circ} \mathrm{C}$ in humid conditions with $5 \% \mathrm{CO}_{2}$. The amount of insulin was determined using the human insulin radioimmunoassay kit (RIA kit, Millipore, Burlington, MA, USA).

\section{Transplantation in mouse model}

Male GalT KO mice weight 20 to $30 \mathrm{~g}$ were used as recipients for each type microencapsulated islet transplantation. Their presence of diabetes was considered if two or more non-fasting blood glucose levels from the tail vein exceed $300 \mathrm{mg} / \mathrm{dL}$ after intraperitoneal injection of STZ. Two groups of transplanted animals were studied: (1) alginate microencapsulated islets were transplanted (group 1; $n=5$ ); (2) Dexa-chitosan-coated alginate microencapsulated islets were transplanted (group $2 ; n=6$ ). Microencapsulated islets (10,000 IEq) were slowly infused into the peritoneal cavity via a flank incision. After transplantation, blood glucose levels were monitored every day in the first week and twice a week afterwards. Graft failure was determined if three consecutive non-fasting blood glucose levels exceeds 300 $\mathrm{mg} / \mathrm{dL}$.

\section{Fibrosis score calculate}

Two hundred and thirty-one days after transplantation, microcapsules were harvested under ketamine $\mathrm{HCl}$ anesthesia by peritoneal lavage using warm $1 \times$ HBSS. The harvested microcapsules were stained with DTZ and assessed under an inverted microscope to establish the degree of fibrotic cell infiltration (CKX41, Olympus, Tokyo, Japan). Images were captured by an E330 digital camera (Olympus) connected to the inverted microscope. Fibrosis infiltration score was defined on the harvested microcapsule surface (Fig. 1) [27]. Fibrosis infiltration score $=(0 \times 0 \% /$ total $)+(3.3 \times<50 \% /$ total $)+(6.6 \times>50 \% /$ total $)+(10 \times$ $100 \% /$ total).

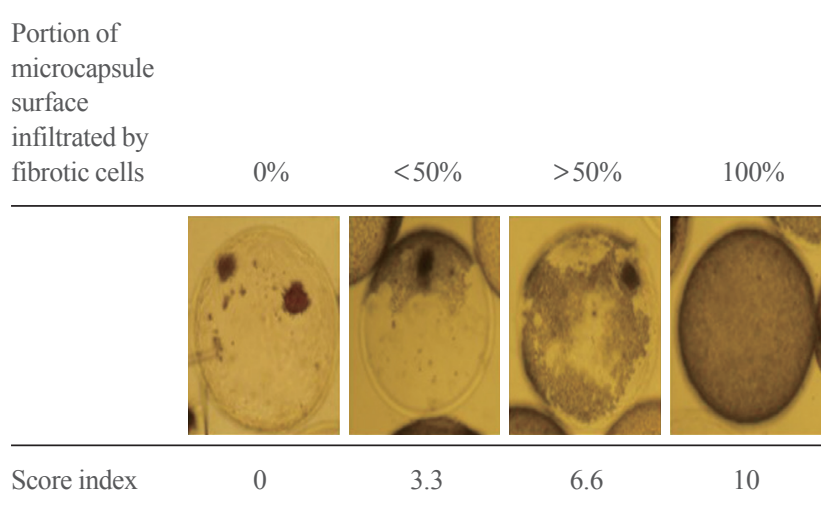

Fig. 1. Classification of microcapsules harvested after graft failure in the peritoneal cavity of galactosyltransferase knock-out mice mice based on the extent of fibrotic cell infiltration to the microcapsule surface. 


\section{Immunohistochemistry and Masson-Trichrome stain}

Harvested microcapsules were fixed 15 to 30 minutes in $4 \%$ PFA at $4^{\circ} \mathrm{C}$. Fixed capsules were embedded with 5\% LB agarose at room temperature for 10 minutes. And, it was sectioned at 4 to $7 \mu \mathrm{m}$. Immunohistochemistry staining was conducted using specific antibodies such as insulin, macrophage, CD3. Specimens were incubated overnight at $4^{\circ} \mathrm{C}$ with diluted primary antibodies (insulin, CD3, macrophage; Abcam, Cambridge, MA, USA), and then incubated for 1 hour with fluorescence-conjugated secondary antibodies (FITC, Rhodamine, Vector Lab, Burlingame, CA, USA) at room temperature. DAPI (Sigma-Aldrich) was employed for nuclear staining. Immunofluorescence was detected under a fluorescence microscope (Observer.Z1). For infiltrated fibrosis on harvested microcapsules stain, deparaffinized specimens were incubated with weigert's iron hematoxylin at room temperature for 10 minutes. Next, Biebrich scarlet-acid fuchin solution at 15 minutes and 5\% aqueous phosphotungstic acid at 3 minutes and $1 \%$ glacial acetic acid at 3 minutes. Finally, it was incubated with light green solution at 15 minutes.

\section{Statistical analysis}

Results were presented as the mean \pm standard error of mean values. Survival rate was analyzed using log-rank test survival curves, differences between two groups were analyzed using the two-tailed unpaired $t$ test using GraphPad Prism version 5.0 (GraphPad software Inc., La Jolla, CA, USA). $P$ value of less than 0.05 was assumed statistical significance.

\section{RESULTS}

\section{Characterization of Dexa-chitosan-coated alginate microcapsules}

The layer of Dexa-chitosan coating on alginate microcapsules was observed by TEM (Fig. 2A). The permeability of each microcapsule was estimated using a diffusion assay of FITC-labeled dextran molecules of various sizes (Fig. 2B). Dexa-chitosan-coated alginate microcapsules exhibited similar diffusion of FITC-dextran at a molecular weight of 4 to $40 \mathrm{kDa}$ compared with alginate microcapsules. This finding suggests that the Dexa-chitosan coating did not affect the permeability of gas exchange and nutrients through the microcapsule inner core. However, diffusion of FITC-labeled dextran in Dexa-chitosan-coated alginate microcapsules was blocked between 40 and $70 \mathrm{kDa}$. This finding suggests that the molecular cutoff weight of the capsule membrane is at least $70 \mathrm{kDa}$, which can prevent im- mune cells and immunoglobulin $\mathrm{G}(\mathrm{IgG} ; 150 \mathrm{kDa})$ from penetrating into the microcapsules. Dexa release from Dexa-chitosan-coated alginate microcapsules was measured using a UV/ Vis spectrometer (Fig. 2C). Dexa release was relatively constant over 35 days after microencapsulation.

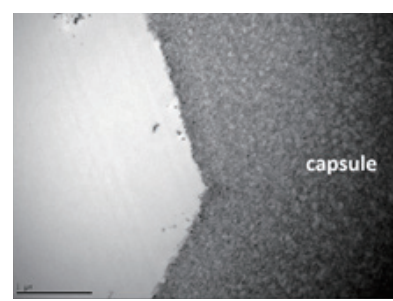

Alginate microcapsule

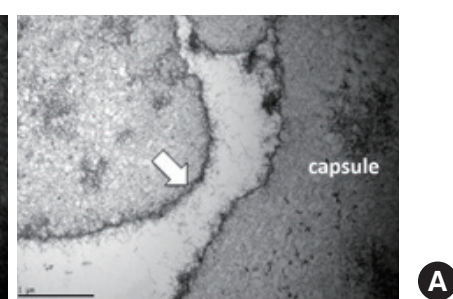

Dexa-chitosan-coated alginate microcapsule

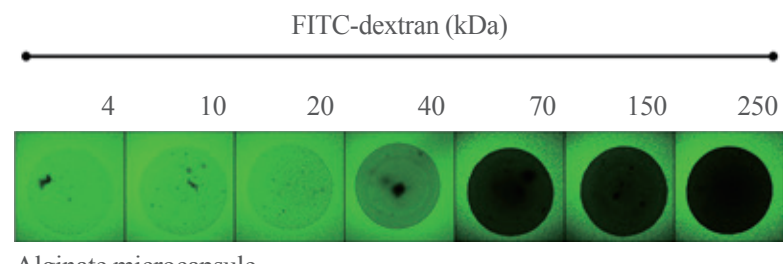

Alginate microcapsule

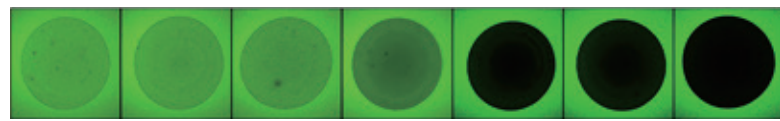

Dexa-chitosan-coated alginate microcapsule

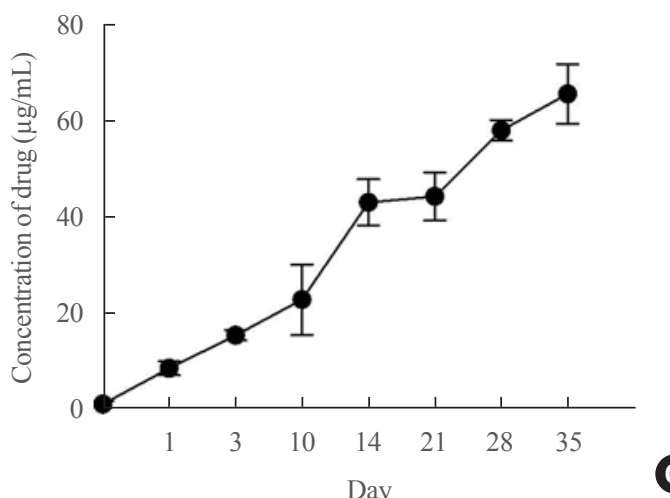

Fig. 2. Characterization of dexamethasone (Dexa)-chitosan-coated alginate microcapsules. (A) Observation of the microcapsule surface by transmission electron microscopy. The left panel is the alginate microcapsule $(\times 10,000$ magnification), and the right panel is the Dexa-chitosan-coated alginate microcapsule (white arrow; dexa-chitosan layer). (B) Observation of the diffusion and permeability assessment of microcapsules by fluorescein isothiocyanate (FITC)-labeled dextran. The molecular cutoff of each microcapsule type was between 40 and $70 \mathrm{kDa}$. (C) Dexa concentration for 36 days after incubation of 1,000 ea Dexa-chitosan-coated alginate microcapsules. 

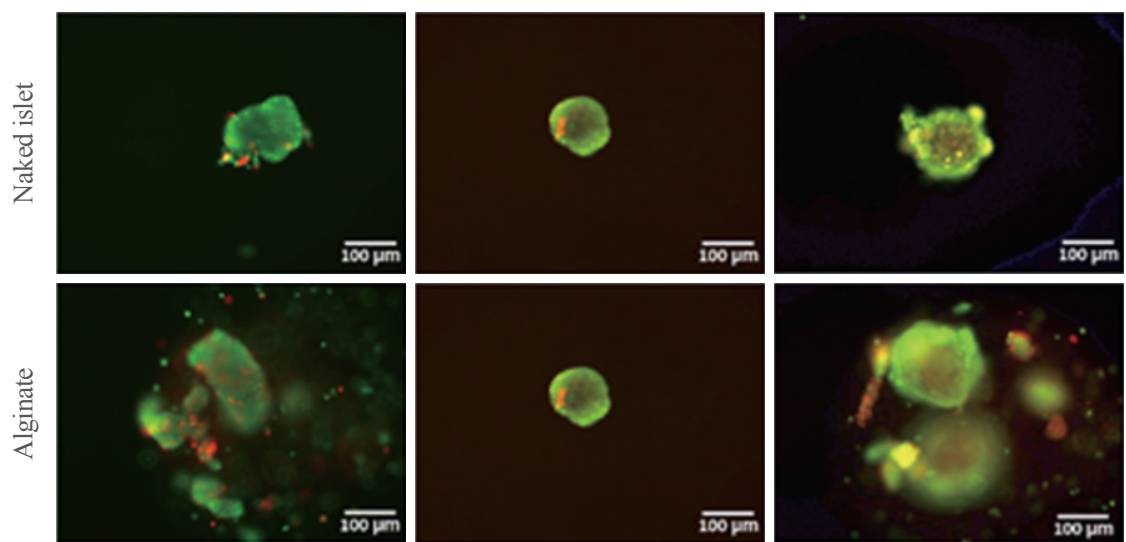

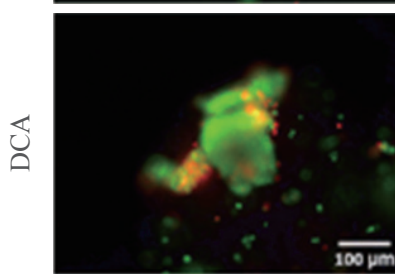

Day 0

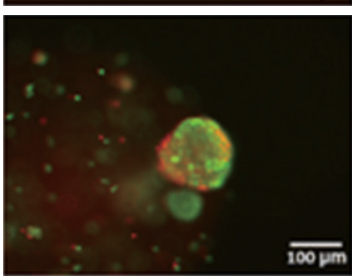

Day 3

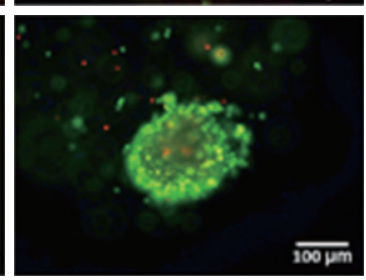

Day 7
A
TIII Naked islets

Alginate encapsulation islets

Dexa-chitosan-coated alginate encapsulation islets

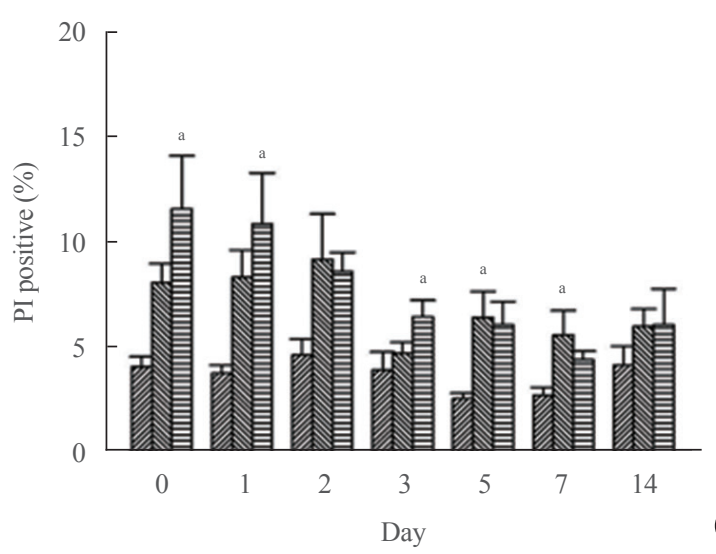

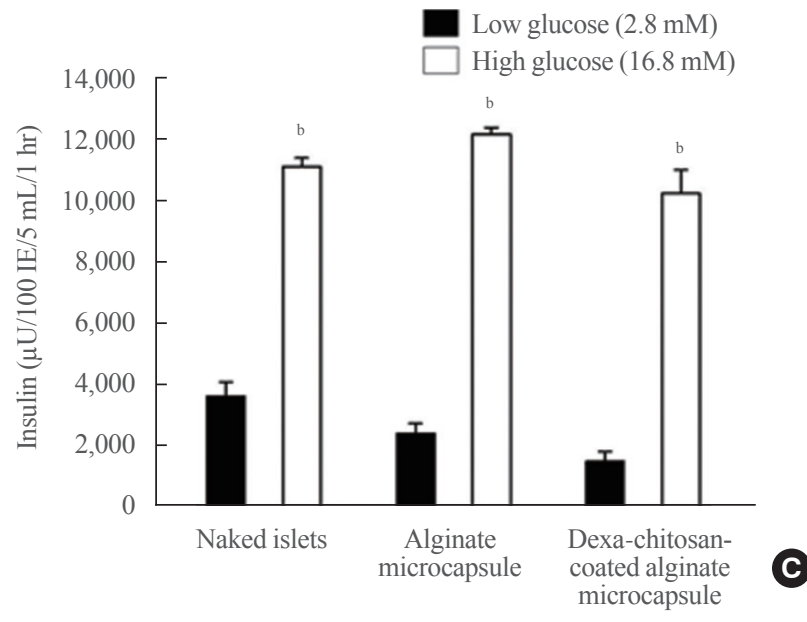

Fig. 3. Viability and secretory function of the microencapsulation islets. (A) Viability of microencapsulated islets based on acridine orange/ propidium iodide $(\mathrm{AO} / \mathrm{PI})$ staining of naked islets, alginate microcapsules, and dexamethasone (Dexa)-chitosan-coated alginate microcapsules (DCAs) at days 0, 3, and 7. Scale bar $=100 \mu \mathrm{m}$. (B) PI-positive cells (\%) in naked islets, alginate microencapsulated islets and Dexachitosan-coated alginate microencapsulated islets over 14 days. (C) Insulin secretory function of microencapsulated islets during glucosestimulated insulin secretion. Insulin secretion was significantly increased in response to glucose in all groups. ${ }^{a} P<0.05$ compared with the naked islets in each date group; ${ }^{\mathrm{b}} P<0.01$.

\section{Viability and function of microencapsulated islets}

The viability of encapsulated islets was confirmed by AO/PI assay on days 0,3 , and 7 after microencapsulation and compared with naked islet viability (Fig. 3A). The survival rate of microencapsulated islets stabilized after 3 days. Fourteen days after microencapsulation, the percentage of PI-positive cells in the Dexa-chitosan-coated alginate group $(6.05 \% \pm 1.73 \%)$ was sim- ilar to that of the alginate group $(5.99 \% \pm 0.80 \%)$ and did not differ from that of the naked islets $(4.13 \% \pm 0.91 \%)$ (Fig. 3B). Islet function assessed by GSIS was also similar among naked, alginate, and Dexa-chitosan-coated alginate groups 14 days after microencapsulation. These results suggest that islet viability and function were well maintained after microencapsulation (Fig. 3C). 

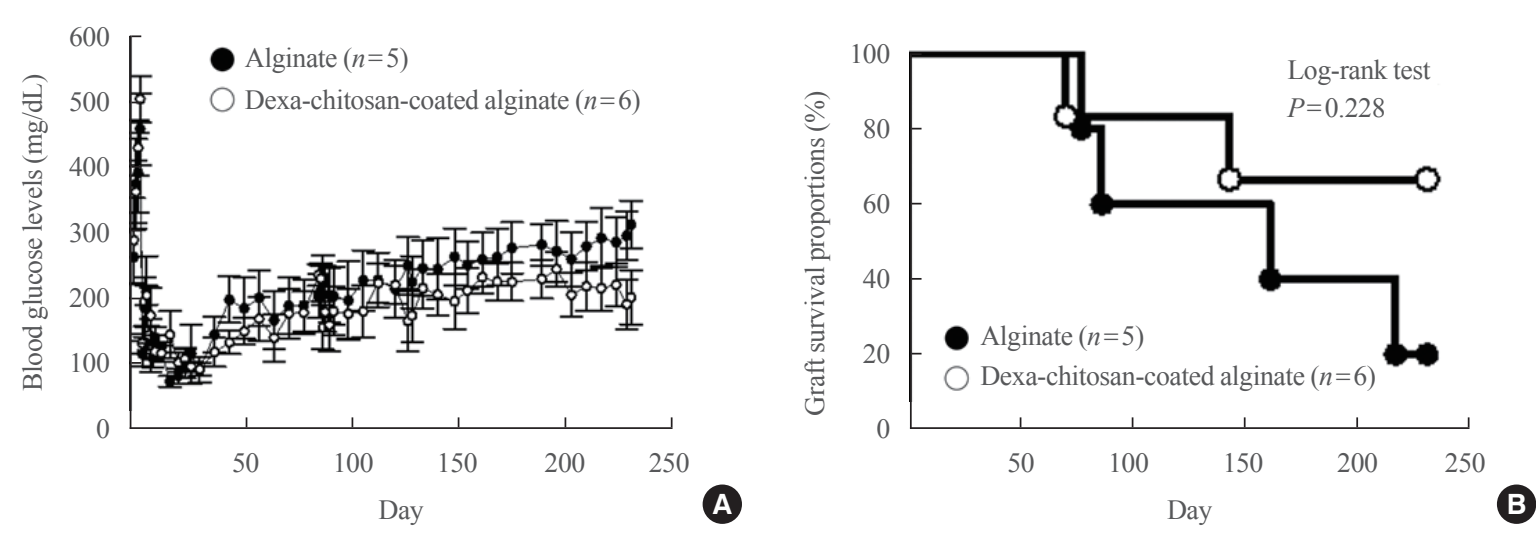

Fig. 4. Blood glucose level and graft survival in galactosyltransferase knock-out mice (GalT KO) mice transplanted with microencapsulated islets. (A) Change in blood glucose levels in GalT KO mice transplanted with alginate microencapsulated islets $(n=5)$ and dexamethasone (Dexa)-chitosan coated alginate microencapsulated islets $(n=6)$. (B) Graft survival proportions in GalT KO mice transplanted with microencapsulated islets. Graft survival proportions of Dexa-chitosan-coated alginate microencapsulated islets (open circle) improved approximately $60 \%$ compared with $20 \%$ survival for alginate microencapsulated islets (close circle) (log-rank test, $P=0.228$ ).

\section{Improvement of blood glucose levels after Dexa-chitosan- coated alginate microencapsulated islet transplantation in diabetic mice}

Before transplantation, the nonfasting blood glucose levels of all diabetic mice were greater than $300 \mathrm{mg} / \mathrm{dL}$. Blood glucose levels significantly decreased after transplantation and remained within the normal range until 231 days after transplantation in both the Dexa-chitosan-coated alginate and alginate groups (Fig. 4A). The average nonfasting blood glucose level for 231 days after transplantation was significantly reduced in the Dexa-chitosan-coated alginate group compared with the alginate group $(176.0 \mathrm{mg} / \mathrm{dL}$ vs. $202.7 \mathrm{mg} / \mathrm{dL}, P<0.05)$. The graft survival rate was higher in the Dexa-chitosan alginate group compared with the alginate group, but the difference was not significant (log rank test, $P=0.228$ ) (Fig. $4 \mathrm{~B}$ ).

\section{Improved fibrosis of Dexa-chitosan-coated alginate microcapsules after transplantation}

Pericapsular fibrosis was observed from harvested microcapsules 231 days after transplantation. As shown in Fig. 5A (upper panels), the fibrosis of Dexa-chitosan-coated alginate microencapsulated islets was conspicuously decreased compared with that of the alginate group. The fibrosis infiltration score was significantly reduced in the Dexa-chitosan-coated alginate group compared with the alginate group (Fig. 5B). Similarly, Masson's trichrome staining revealed significant collagen deposition around alginate microcapsules but not around Dexa-chitosancoated alginate microcapsules (Fig. 6A). In alginate microcapsules, most infiltrated cells were positive for macrophage stain- ing, and a few cells were positive for CD4 staining (Fig. 6B). In contrast, inflammatory cell infiltration was not observed in Dexa-chitosan-coated alginate microcapsules (Fig. 6B). Taken together, these results suggest that Dexa-chitosan-coated alginate microcapsules can significantly suppress pericapsular fibrosis and inflammatory cell infiltration in xenogeneic microencapsulated islet transplantation compared with the alginate group.

The graft function of harvested microencapsulated islets was assessed by DTZ and insulin staining. In the Dexa-chitosancoated alginate group, most harvested cells were positive for DTZ and insulin (Fig. 5A, lower panel and Fig. 6C). Although alginate microcapsules exhibited significant pericapsular fibrosis, DTZ and insulin staining were positive in most harvested cells (Fig. 5A, lower panel and Fig. 6C).

\section{DISCUSSION}

In this study, we developed Dexa-chitosan-coated alginate microcapsules that have similar properties (capsular pore size, islet viability, and insulin secretion) to alginate microcapsules but improved biocompatibility. After xenogeneic transplantation, blood glucose levels were normalized in both groups, but the mean glucose level was lower in the Dexa-chitosan group compared with the alginate group. The graft survival rate was higher in the Dexa-chitosan group, but not significantly, compared with the alginate group. Pericapsular fibrosis and inflammatory cell infiltration of harvested microcapsules were significantly reduced in Dexa-chitosan-coated alginate microcapsules com- 

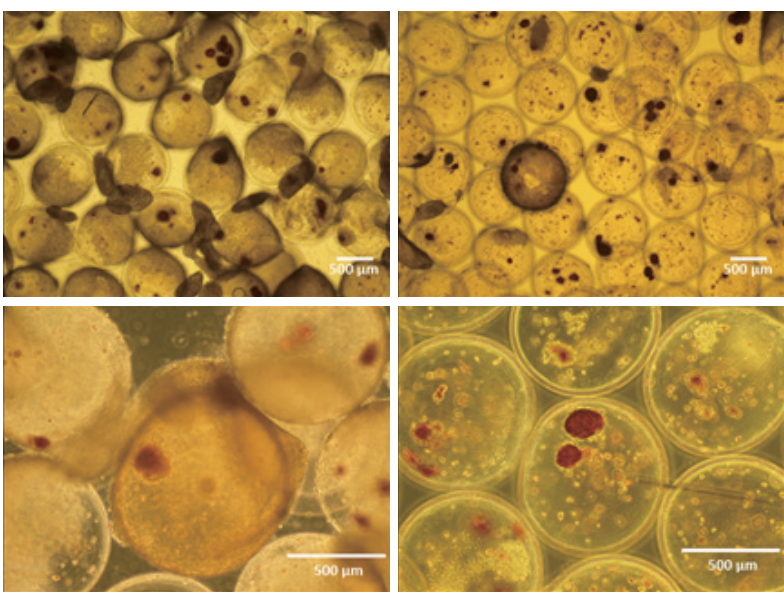

Alginate microcapsule

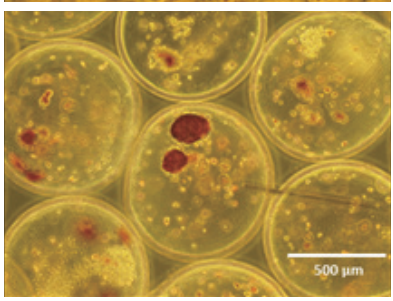

Dexa-chitosan-coated alginate microcapsule

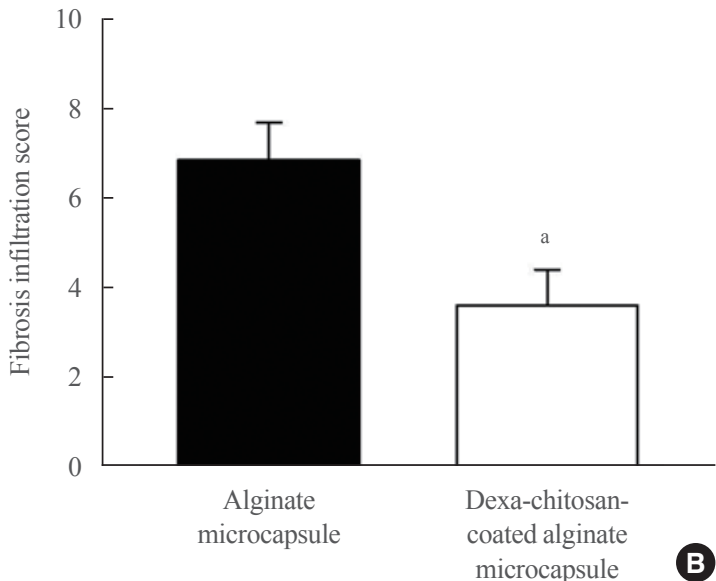

A

Fig. 5. Dithizone staining of harvested microencapsulated porcine islets and the measurement of fibrotic cell infiltration to the microcapsule surface. (A) The left panel shows harvested porcine islets with alginate microcapsules, and the right panel shows harvested dexamethasone (Dexa)-chitosan-coated alginate microcapsules. (B) Fibrosis infiltration score of microcapsules compared with alginate- and Dexa-chitosancoated alginate microcapsules at 231 days after 10,000 IEq islet transplantation. ${ }^{a} P<0.05$ compared with alginate microcapsules.
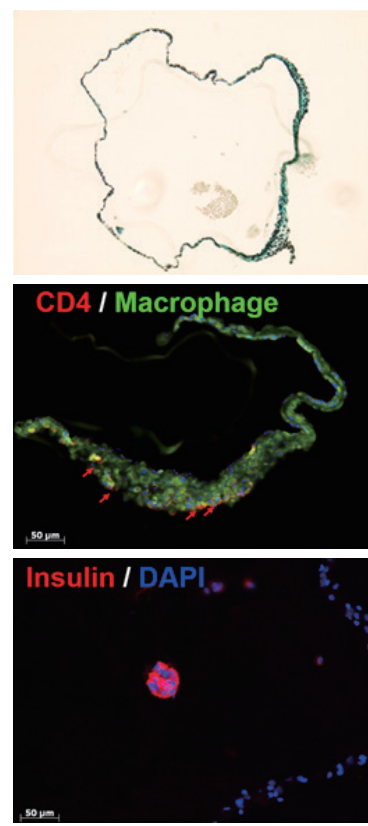

Alginate microcapsule
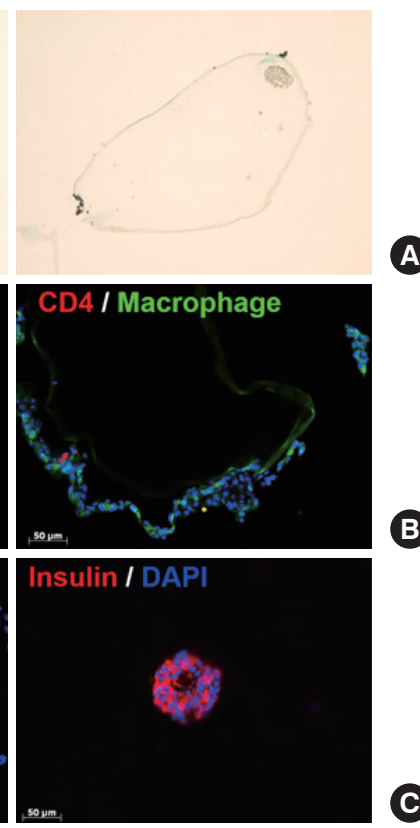

Dexa-chitosan-coated alginate microcapsule

Fig. 6. Trichrome or immunohistochemistry staining of harvested encapsulated porcine islets and measurement of fibrotic cell infiltration to the microcapsule surface. (A) Fibrosis infiltrated stain (green) harvested from alginate microcapsules and dexamethasone (Dexa)chitosan-coated alginate microcapsules. (B) Macrophages (green) and CD3 (red) were stained in alginate microcapsules. Red arrows indicate CD3-positive cells. Scale bar $=50 \mu \mathrm{m}$. (C) Porcine islets were stained with insulin antibody (red). Scale bar=50 $\mu \mathrm{m}$. DAPI, 4'-6-diamidino-2-phenylindole.

pared with alginate microcapsules.

The microcapsule has been studied to avoid the immune rejection response from various immune cells, i.e., immunoglobulin and cytochemokine-induced pathways [28,29]. We previously reported that alginate microencapsulated islets improve blood glucose levels without immunosuppressants in diabetic mice [30]. However, alginate microcapsules have a limitation of pervasive fibrosis in the pericapsular area. The application of antiinflammatory regimens has the potential to alleviate the immunological response to transplanted medical devices [31] or implanted cell-based therapeutics [32,33]. Dang et al. [34] assessed the immunosuppressive effects of 16 small molecules on the host response to transplanted biomaterials by subcutaneous injection. Among them, Dexa and curcumin effectively suppress early inflammation markers, such as reactive oxygen species and cathepsin activity [34]. In particular, Dexa exhibits a superior reduction in cathepsin activity [34]. Therefore, this study sought to overcome pericapsular inflammation and fibrosis with Dexa-chitosan coating on alginate microcapsules in xenotransplantation.

We previously developed a rapamycin-alginate capsule to improve the biocompatibility of encapsulated islets [35]. Rapamycin-polyethylene glycol-coated alginate microcapsules strongly inhibit fibrosis on the microcapsule surface. However, rapamycin has a limitation that impairs islet function. In contrast to a previous study, the current study demonstrated that Dexa-chitosan-coated alginate microcapsules inhibited inflammatory cell 
infiltration and fibrosis while preserving islet function. Another study reported improved pericapsular fibrosis and islet viability in alginate microcapsules coated with pentoxifylline, which is known to inhibit the inflammatory response induced by interleukin-2 and tumor necrosis factor- $\alpha$ [36]. However, this finding was only demonstrated in vitro experiments. Therefore, the effects of pentoxifylline-coated alginate capsules on islet function and inflammation should be assessed in an in vivo transplantation model. We demonstrated preserved islet function and improved fibrosis and inflammatory cell infiltration of Dexa-chitosan-coated alginate microcapsules in vitro and in vivo.

Both Dexa-chitosan-coated alginate and alginate microcapsules have the same molecular cutoff weight of $70 \mathrm{kDa}$. This finding suggests that it can effectively block the penetration of high molecular weight molecules, such as macrophages and IgG, but not small molecules, such as cytokines. The immune response caused by these small molecules induces inflammation, fibrosis and islet damage. In our study, despite improved blood glucose levels, alginate microcapsules showed significant fibrosis and inflammatory cell infiltration on the capsular surface, which could be critical for long-term graft survival and clinical application of transplantation. Meanwhile, these reactions were rarely observed in Dexa-chitosan-coated alginate microcapsules. Our findings suggest that Dexa coating on microcapsules could improve biocompatibility through control of the immune response and increase the potential for clinical application of encapsulated islet transplantation.

In conclusion, Dexa coating on alginate microcapsules significantly suppressed pericapsular fibrosis and inflammatory cell infiltration in microencapsulated islet transplantation. These results suggest that Dexa-chitosan-coated alginate microcapsules potentially represent a promising technology for both xenogeneic islet transplantation. This could also be used for allogeneic islet transplantation that has less immune response than xenogeneic islet transplantation

\section{CONFLICTS OF INTEREST}

No potential conflict of interest relevant to this article was reported.

\section{ACKNOWLEDGMENTS}

This research was supported by Basic Science Research Program through the National Research Foundation of Korea (NRF) funded by the Ministry of Education (NRF-2019R1I-
1A1A01059818), Cooperative Research Program for Agriculture Science and Technology Development Rural Development Administration, Republic of Korea (PJ01345301), and a grant of the Korea Health Technology R\&D Project through the Korea Health Industry Development Institute (KHIDI) funded by the Ministry of Health \&Welfare, Republic of Korea (grant number: HI13C0954).

\section{AUTHOR CONTRIBUTIONS}

Conception or design: H.S.P., K.H.Y. Acquisition, analysis, or interpretation of data: M.J.K., H.S.P., J.W.K., E.Y.L., M.R., Y.H.Y. Drafting the work or revising: M.J.K., H.S.P., J.W.K., E.Y.L. Final approval of the manuscript: G.K., C.G.P., K.H.Y.

\section{ORCID}

Min Jung Kim https://orcid.org/0000-0003-0667-6290

Kun-Ho Yoon https://orcid.org/0000-0002-9109-2208

\section{REFERENCES}

1. Atkinson MA, Eisenbarth GS. Type 1 diabetes: new perspectives on disease pathogenesis and treatment. Lancet 2001;358:221-9.

2. Lipsett M, Aikin R, Castellarin M, Hanley S, Jamal AM, Laganiere $\mathrm{S}$, et al. Islet neogenesis: a potential therapeutic tool in type 1 diabetes. Int J Biochem Cell Biol 2006;38:498503.

3. Murdoch TB, McGhee-Wilson D, Shapiro AM, Lakey JR. Methods of human islet culture for transplantation. Cell Transplant 2004;13:605-17.

4. Sakata N, Sumi S, Yoshimatsu G, Goto M, Egawa S, Unno M. Encapsulated islets transplantation: past, present and future. World J Gastrointest Pathophysiol 2012;3:19-26.

5. Boker A, Rothenberg L, Hernandez C, Kenyon NS, Ricordi C, Alejandro R. Human islet transplantation: update. World J Surg 2001;25:481-6.

6. Shapiro AM, Lakey JR, Ryan EA, Korbutt GS, Toth E, Warnock GL, et al. Islet transplantation in seven patients with type 1 diabetes mellitus using a glucocorticoid-free immunosuppressive regimen. N Engl J Med 2000;343:230-8.

7. Collaborative Islet Transplant Registry. CITR Tenth Annual Reports [Internet]. Rockville: citr; 2017 [cited 2020 Nov 25]. Available from: http://citregistry.org/content/reports-publications-presentations. 
8. Larsen JL, Bennett RG, Burkman T, Ramirez AL, Yamamoto $\mathrm{S}$, Gulizia J, et al. Tacrolimus and sirolimus cause insulin resistance in normal Sprague Dawley rats. Transplantation 2006;82:466-70.

9. Dai C, Walker JT, Shostak A, Padgett A, Spears E, Wisniewski $\mathrm{S}$, et al. Tacrolimus- and sirolimus-induced human $\beta$ cell dysfunction is reversible and preventable. JCI Insight 2020;5:e130770.

10. Dufrane D, Gianello P. Macro- or microencapsulation of pig islets to cure type 1 diabetes. World J Gastroenterol 2012; 18:6885-93.

11. Juste S, Lessard M, Henley N, Menard M, Halle JP. Effect of poly-L-lysine coating on macrophage activation by alginate-based microcapsules: assessment using a new in vitro method. J Biomed Mater Res A 2005;72:389-98.

12. Lim DJ, Cho JH, Choi YH, Park HS, Hong OK, Kwon HS, et al. Optimization of microencapsulation of the Islets. Tissue Eng Regen Med 2005;2:280-6.

13. Jiang K, Weaver JD, Li Y, Chen X, Liang J, Stabler CL. Local release of dexamethasone from macroporous scaffolds accelerates islet transplant engraftment by promotion of anti-inflammatory M2 macrophages. Biomaterials 2017;114: 71-81.

14. Wu P, Grainger DW. Drug/device combinations for local drug therapies and infection prophylaxis. Biomaterials 2006; 27:2450-67.

15. Labhasetwar V, Levy RJ. Implants for site-specific drug delivery. J Appl Biomater 1991;2:211-2.

16. Jones JA, Chang DT, Meyerson H, Colton E, Kwon IK, Matsuda T, et al. Proteomic analysis and quantification of cytokines and chemokines from biomaterial surface-adherent macrophages and foreign body giant cells. J Biomed Mater Res A 2007;83:585-96.

17. Ramalingam A, Hirai A, Thompson EA. Glucocorticoid inhibition of fibroblast proliferation and regulation of the cyclin kinase inhibitor p21Cip1. Mol Endocrinol 1997;11:57786.

18. Shi K, Jiang J, Ma T, Xie J, Duan L, Chen R, et al. Dexamethasone attenuates bleomycin-induced lung fibrosis in mice through TGF- $\beta$, Smad 3 and JAK-STAT pathway. Int J Clin Exp Med 2014;7:2645-50.

19. Zawalich WS, Tesz GJ, Yamazaki H, Zawalich KC, Philbrick W. Dexamethasone suppresses phospholipase C activation and insulin secretion from isolated rat islets. Metabolism 2006;55:35-42.

20. Kaiser G, Gerst F, Michael D, Berchtold S, Friedrich B,
Strutz-Seebohm N, et al. Regulation of forkhead box O1 (FOXO1) by protein kinase B and glucocorticoids: different mechanisms of induction of beta cell death in vitro. Diabetologia 2013;56:1587-95.

21. Kim JW, Sun C, Jeon SY, You YH, Shin JY, Lee SH, et al. Glucocorticoid treatment independently affects expansion and transdifferentiation of porcine neonatal pancreas cell clusters. BMB Rep 2012;45:51-6.

22. Jin SM, Shin JS, Kim KS, Gong CH, Park SK, Kim JS, et al. Islet isolation from adult designated pathogen-free pigs: use of the newer bovine nervous tissue-free enzymes and a revised donor selection strategy would improve the islet graft function. Xenotransplantation 2011;18:369-79.

23. O’Neil JJ, Stegemann JP, Nicholson DT, Gagnon KA, Solomon BA, Mullon CJ. The isolation and function of porcine islets from market weight pigs. Cell Transplant 2001;10: 235-46.

24. Pignatello R, Stancampiano AH, Ventura CA, Puglisi G. Dexamethasone sodium phosphate-loaded Chitosan based delivery systems for buccal application. J Drug Target 2007; 15:603-10

25. Hall KK, Gattas-Asfura KM, Stabler CL. Microencapsulation of islets within alginate/poly(ethylene glycol) gels crosslinked via Staudinger ligation. Acta Biomater 2011;7:614-24.

26. Sylvestre JP, Guy RH, Delgado-Charro MB. In vitro optimization of dexamethasone phosphate delivery by iontophoresis. Phys Ther 2008;88:1177-85.

27. Tam SK, Bilodeau S, Dusseault J, Langlois G, Halle JP, Yahia LH. Biocompatibility and physicochemical characteristics of alginate-polycation microcapsules. Acta Biomater 2011;7:1683-92.

28. Vergani A, D’Addio F, Jurewicz M, Petrelli A, Watanabe T, Liu K, et al. A novel clinically relevant strategy to abrogate autoimmunity and regulate alloimmunity in NOD mice. Diabetes 2010;59:2253-64.

29. Merani S, Shapiro AM. Current status of pancreatic islet transplantation. Clin Sci (Lond) 2006;110:611-25.

30. Park HS, Ham DS, You YH, Shin JY, Kim JW, Jo JH, et al. Successful xenogenic islet transplantation with Ba2+-alginate encapsulation. Tissue Eng Regen Med 2010;7:523-30.

31. Singarayar S, Kistler PM, De Winter C, Mond H. A comparative study of the action of dexamethasone sodium phosphate and dexamethasone acetate in steroid-eluting pacemaker leads. Pacing Clin Electrophysiol 2005;28:311-5.

32. Schneider BL, Schwenter F, Pralong WF, Aebischer P. Prevention of the initial host immuno-inflammatory response 
determines the long-term survival of encapsulated myoblasts genetically engineered for erythropoietin delivery. Mol Ther 2003;7:506-14.

33. Zhang WJ, Marx SK, Laue C, Hyder A, Juergensen A, Bickel M, et al. HOE 077 reduces fibrotic overgrowth around the barium alginate microcapsules. Transplant Proc 2000;32: 206-9.

34. Dang TT, Thai AV, Cohen J, Slosberg JE, Siniakowicz K, Doloff JC, et al. Enhanced function of immuno-isolated islets in diabetes therapy by co-encapsulation with an anti-in- flammatory drug. Biomaterials 2013;34:5792-801.

35. Park HS, Kim JW, Lee SH, Yang HK, Ham DS, Sun CL, et al. Antifibrotic effect of rapamycin containing polyethylene glycol-coated alginate microcapsule in islet xenotransplantation. J Tissue Eng Regen Med 2017;11:1274-84.

36. Azadi SA, Vasheghani-Farahani E, Hashemi-Najafbabadi S, Godini A. Co-encapsulation of pancreatic islets and pentoxifylline in alginate-based microcapsules with enhanced immunosuppressive effects. Prog Biomater 2016;5:101-9. 